\title{
Association Between Iron and Cholesterol in Neuroblastomas
}

\author{
SANG Y. LEE ${ }^{1 *}$, BECKY SLAGLE-WEBB ${ }^{1}$, CARA-LYNNE SCHENGRUND $^{2}$, \\ JUNJIA ZHU ${ }^{3}$ and JAMES R. CONNOR ${ }^{1}$ \\ ${ }^{1}$ Department of Neurosurgery, Pennsylvania State University College of Medicine, Hershey, PA, U.S.A.; \\ ${ }^{2}$ Department of Biochemistry and Molecular Biology, \\ Pennsylvania State University College of Medicine, Hershey, PA, U.S.A.; \\ ${ }^{3}$ Department of Public Health Sciences, Pennsylvania State University College of Medicine, Hershey, PA, U.S.A.
}

\begin{abstract}
Background/Aim: Neuroblastoma is the most common childhood extracranial solid malignancy. Although cancer cells need iron and lipids for active cell division, possible links between iron and lipid metabolism in neuroblastomas have not been studied. Materials and Methods: We evaluated the levels and association between iron and cholesterol on in vitro neuroblastoma cancer models. Results: We found that the levels of iron and cholesterol are diverse among neuroblastoma cell lines. There is a bi-directional association between iron and cholesterol in drug-resistant neuroblastoma SK-N-AS cells. In drug-resistant neuroblastoma cells, low concentration of an iron chelator did not have an impact on iron levels, but on cellular cholesterol levels. Furthermore, a cholesterol decreasing agent, simvastatin, influenced both iron and cholesterol levels in drugresistant neuroblastoma cells. Conclusion: Cholesterol decreasing agents may be more effective than iron chelators for drug-resistant neuroblastoma treatment.
\end{abstract}

Neuroblastoma, the most common extracranial solid tumor in children, accounts for about $7.5 \%$ of all childhood cancers and $\sim 15 \%$ of all pediatric oncology-related deaths $(1,2)$. Approximately $50 \%$ of neuroblastoma patients have metastatic disease and adverse tumor-specific biological features at the time of diagnosis $(3,4)$. The variation seen in neuroblastoma is thought to arise from arrest and deregulation of normal development of the neural crest (5). The two most common

This article is freely accessible online.

*Current address: Department of Neurology, The Johns Hopkins University School of Medicine, Baltimore, MD, U.S.A.

Correspondence to: Dr. Sang Y. Lee, 500 University Drive (H110), Hershey, PA 17033, U.S.A. Tel: +1 7175314541, Fax: +1 7175310091, e-mail: 1sysys627@gmail.com

Key Words: Deferoxamine, drug-resistance, iron chelator, simvastatin. risk factors for neuroblastomas are age and genetic factors. Both the International Staging System (6) and the International Neuroblastoma Risk Group Staging System (7) note that children less than 18 months of age have the best prognosis while those whose tumors have metastasized to distant parts of the body have the poorest outcome when over 18 months of age. Presently, genetic mutations are also used in the staging of neuroblastomas. These can include germline mutations ( 8 , 9) (e.g., ALK, MYCN), or somatic events (10-13) (e.g., somatic mutations, copy number variation, somatic DNA-amplification, somatic chromosomal gains and losses). Most children with neuroblastoma are treated with chemotherapy. Those with highrisk neuroblastomas often receive a combination of drugs as the primary treatment, either prior to and/or after surgery.

Iron is an essential trace metal for normal as well as malignant cancer cells. In general, cancer cells exhibit increased iron dependence, hence increased stores of body iron are thought to be linked to increased cancer risk $(14,15)$, and the cellular iron level is closely associated with cancer characteristics. The proliferation of cancer cells requires large amounts of lipids, the building blocks for the cell's biological membranes. Seven of the enzymes needed for cholesterol synthesis are iron-dependent (16). Interestingly cholesterol accumulation in either blood or the tumor has been documented in patients with prostate, oral, and endometrial cancer (17-20). Studies interrogating the association between iron per se and cholesterol metabolism are limited. There is an excellent review paper for the link between iron and lipids in human neurodegenerative disease (21). Data from a study carried out over an 18-year time span indicated that ironinduced oxidation of serum lipids is important in cancer pathogenesis (22). Mainous $3^{\text {rd }}$ et al. also reported that high iron levels raise the risk of cancer in people with high cholesterol (23). However, there appear to be no reports describing a relationship between iron and cholesterol metabolism in childhood cancers. To address this question, we investigated the relationship between iron and cholesterol in neuroblastoma cells using an in vitro cell culture model. The 
overreaching hypothesis behind this work is that the identification of a unique relationship between iron and cholesterol in treatment-resistant neuroblastomas could provide the basis for the development of new treatment options.

\section{Materials and Methods}

Materials. All cell culture reagents including Dulbecco's Modified Eagle's Medium/Nutrient Mixture F-12 (DMEM/F12, cat \#11330057, lot \#1750072) and Iscove's Modified Dulbecco's Medium (IMDM, cat \#12440-079, lot \#1663817) were purchased from Life Technologies (Grand Island, NY, USA). Fetal bovine serum (FBS, cat \#100-106, lot \#A27D00D) was ordered from Gemini Bio-Products (West Sacramento, CA, USA) and lipoprotein-deficient serum was ordered from Sigma-Aldrich (cat \#S5394, lot \#SLBL5464V) (St. Louis, MO, USA). Deferoxamine (DFO) (cat \#D9533), di-2pyridylketone-4-cyclohexyl-4-methyl-3-thiosemicarbazone (DpC) (cat \#SML0483, lot \#092M4615V), Di-2-pyridylketone-4,4-dimethyl-3thiosemicarbazone (Dp44mT) (cat \#SML0186), U18666A (cat \#U3633, lot \#023M4603V), and simvastatin (cat \#S6196, lot \#053M4728V) were purchased from Sigma-Aldrich. Ezetimibe (cat \#S1655) and Triapine (cat \#S7470) were obtained from Selleckchem (Houston, TX, USA). Stock concentrations of chemicals were prepared in sterile $\mathrm{ddH}_{2} \mathrm{O}$ or DMSO (Sigma, cat \#D2650, lot \#RNBD6333) and appropriately diluted as needed.

Cell culture. Human neuroblastoma cell lines (SH-SY5Y, SK-N-AS) were purchased from the American Type Culture Collection (ATCC) (Manassas, VA, USA). Drug-resistant human neuroblastoma cell lines (CHLA-136, CHLA-171), established from tumors of patients who were at various stages of therapy $(24,25)$, were obtained from the Children's Oncology Group. Authentication of human neuroblastoma cell lines was performed by Genetica (Burlington, NC, USA) via STR DNA profiling. Drug-resistant neuroblastomas are the cell lines with LC90 values (drug concentration lethal for $90 \%$ of treated cells) greater than the clinically achievable drug levels for at least two of the four agents commonly used in neuroblastoma therapy (melphalan, carboplatin, etoposide, vincristine) (25). Cells were maintained at low passage when possible and new cultures started after either 2-3 months or when cell passage number exceeded ten. Neuroblastoma cells were cultured in DMEM/F12 with $10 \%$ FBS, $1 \%$ antibiotics (Penn-Strep, Life Technologies, cat \#15140-122, lot \#1751324), and $1 \%$ non-essential amino acids (Life Technologies, cat \#M7145, lot \#RNBD2211). Acquired drug-resistant neuroblastoma cells (CHLA136, CHLA-171) were cultured in Iscove's Modified Dulbecco's Medium containing $10 \%$ or $20 \%$ FBS (for experimental studies and cell maintenance, respectively), $4 \mathrm{mM}$ L-glutamine (Life Technologies, cat \#25030081), $5 \mu \mathrm{g} / \mathrm{ml}$ insulin, $5 \mu \mathrm{g} / \mathrm{ml}$ transferrin, and $5 \mathrm{ng} / \mathrm{ml}$ selenous acid (Life Technologies, cat \#41400045 - 100X Insulin-Transferrin-Selenium)

Measurement of iron and cholesterol content. Human neuroblastoma cells were cultured for 1-3 days in 6 well plates with complete cell culture medium or lipoprotein-deficient serum-containing medium for iron measurements. Intracellular iron concentration was determined on cell extracts prepared from $1 \times 10^{5}$ cells using the QuantiChrom Iron Assay Kit (BioAssay Systems, cat \#DIFF-250, lot \#BE11A11) (Hayward, CA, USA) following the manufacturer's instructions (26). To determine cholesterol content, neuroblastoma cells were cultured for 1-3 days in 6 well plates in medium containing lipoprotein deficient serum. Biovision's cholesterol assay kit (cat \#K603-100, lot \#1A280603) (Milpitas, CA, USA) was used to determine the cholesterol content of homogenates of $1 \times 10^{6}$ cells, as previously described (27).

Association between iron and cholesterol. To determine the association between iron and cholesterol, SK-N-AS and CHLA-171 cells cultured in lipoprotein deficient medium were treated with the iron chelator DFO, as performed previously (28), before monitoring cholesterol concentration as well as iron level. To determine the effect of a reduction in cholesterol concentration on the iron level in SK-N-AS and CHLA-171 cells, cells cultured in lipoprotein deficient medium were treated with simvastatin, a 3-hydroxy-3methylglutaryl-CoA (HMGCoA) reductase inhibitor (29), and then both iron level and cholesterol concentration were determined.

Cytotoxic effects of iron chelators and cholesterol decreasing agents on neuroblastoma cells. Human neuroblastoma cells (1-2×104/well) were treated with either different concentrations of an iron chelator (DFO, Dp44mT, triapine, DpC) (30), a HMG-CoA reductase inhibitor (simvastatin) (29), the desmosterol $\Delta 24$-reductase inhibitor (U18666A) (31), or a cholesterol absorption inhibitor (ezetimibe) (32) for $48 \mathrm{~h}$ in cell culture medium (for iron chelators) or medium containing lipoprotein deficient serum (for cholesterol decreasing agents). Reduced FBS was used because the flux of cell cholesterol is linked to the type and concentration of lipoproteins in serum as well as transport proteins expressed by the cells $(33,34)$. Cell cytotoxicity was determined using the alamarBlue assay (InVitrogen, Cat \#DAL700, Lot \#559787) (Grand Island, NY, USA,). The EC50s for each chemical treatment were ascertained using GraphPad Prism software (version 7).

Statistical analysis. Treatment data are shown as the percentage of vehicle-treated control cells. Each experiment was repeated at least three times with 3-6 replicates. For each treatment concentration, the level of iron and cholesterol in the two groups was compared using the two-sample Student's $t$-test or two-way ANOVA for comparison of multiple samples. Data were displayed as mean \pm standard error of the mean (SEM). Two-way ANOVA models were used to analyze data obtained from in vitro cytotoxicity experiments. Group differences in cell viability were examined at each concentration by slicing the group-concentration interactions. All analyses were performed using statistical software SAS version 9.4 (SAS Institute, Cary, NC, USA) or GraphPad Prism 7. Differences among means are considered statistically significant when the $p$-value is less than 0.05 .

\section{Results}

Iron and cholesterol levels of neuroblastoma cells. Iron and cholesterol levels were determined for representative samples of human neuroblastoma cells. At day 2, iron levels in the drug-sensitive SH-SY5Y cells and drug-resistant SK-N-AS cells were higher compared to drug-resistant CHLA-136 and CHLA-171 neuroblastoma cells (Figure 1A). The cholesterol levels in the CHLA-171 cells were significantly higher than other tested neuroblastoma cells (Figure 1A). During the cell culture times, iron levels increased, but cholesterol levels decreased (Figure 1B-D). 
A

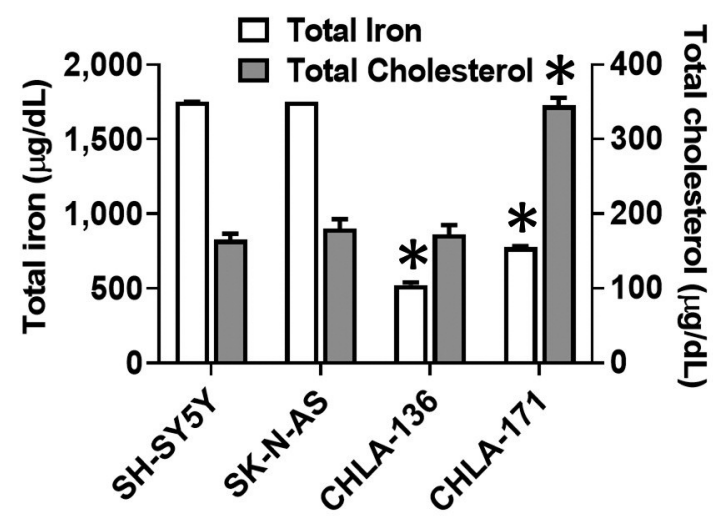

C

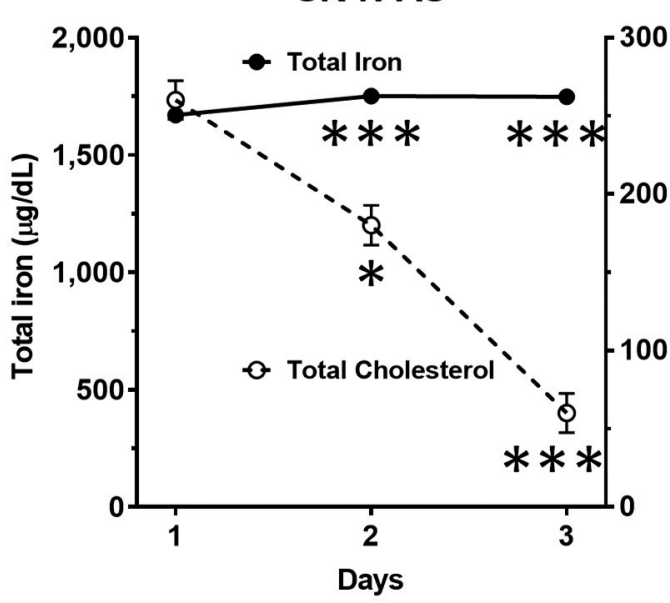

B SH-SY5Y

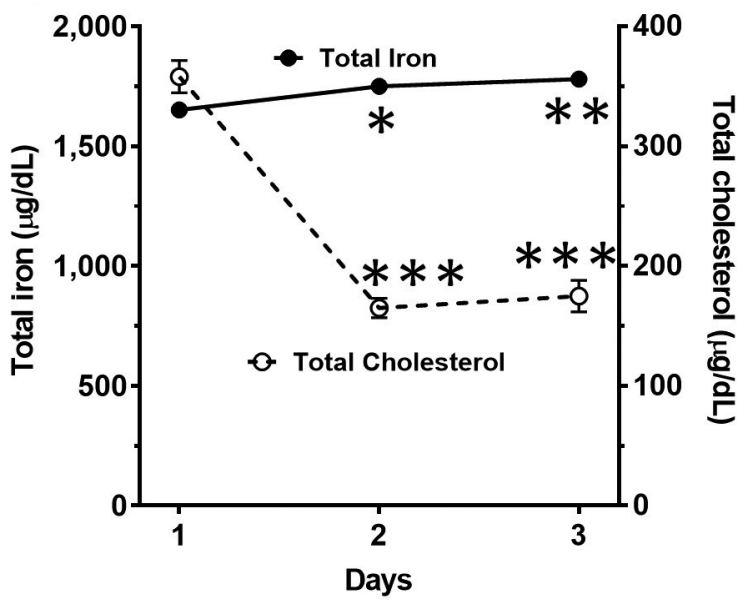

D CHLA-171

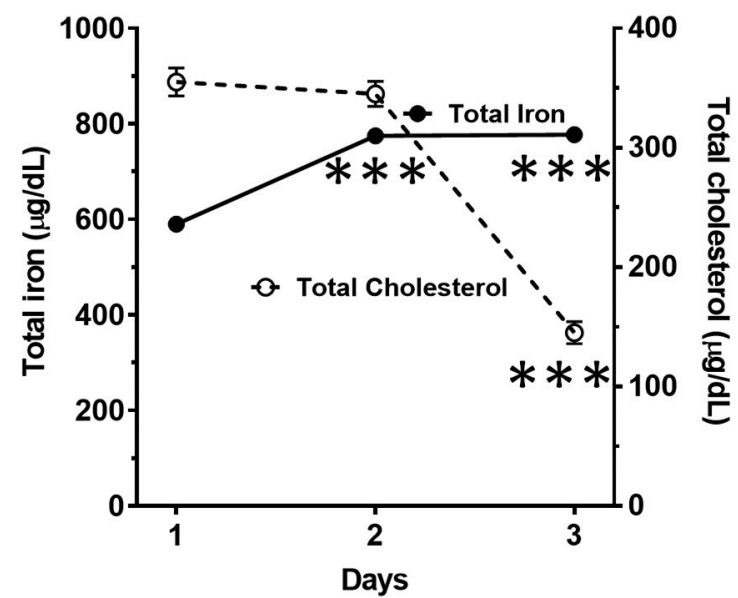

Figure 1. Iron and cholesterol content of human neuroblastoma cells. (A) Human neuroblastoma cells were cultured in complete cell culture medium for 2 days for iron measurements. Intracellular iron concentrations were determined using the QuantiChrom Iron Assay Kit. To determine cholesterol content, neuroblastoma cells were cultured for 2 days in medium containing lipoprotein-deficient serum. Intracellular cholesterol content was determined for $1 \times 10^{6}$ cells using the Biovision cholesterol assay kit. Thirty $\mu \mathrm{g}$ of cell protein extract were used for the iron assay, and $50 \mu \mathrm{l}$ from a total of $200 \mu \mathrm{l}$ of the extracted sample were used for the cholesterol assay. Concentrations of total iron or cholesterol are given in $\mu \mathrm{g} / \mathrm{dl}$. Results represent the mean of three independent experiments with error bars corresponding to standard error. In the case of small errors, bars may not be visible. Asterisks indicate a significant difference compared to the iron or cholesterol level of SH-SY5Y cells $\left(*^{*}<0.05\right)$. (B-D) The cells were cultured for 3 days and then the iron and cholesterol content were determined as described above. Asterisks indicate a significant difference compared to the iron or cholesterol level on day $1 .{ }^{*} p<0.05, * * p<0.01, * * * p<0.001$.

Association between iron and cholesterol in neuroblastoma cells. To determine the possible association between cellular iron and cholesterol concentrations, drug-resistant SK-N-AS and CHLA-171 cells were treated with either the iron chelator DFO or simvastatin in lipoprotein-deficient serumcontaining medium prior to monitoring their iron or cholesterol concentration. In both cells (SK-N-AS, CHLA171), DFO decreased cholesterol levels, although an alteration in iron level was not seen (Figure 2A-B, E-F).
Simvastatin treatment affected both cholesterol and iron levels in the SK-N-AS cells (Figure 2C-D). Unexpectedly, treatment of CHLA-171 cells with $2.5 \mu \mathrm{M}$ simvastatin resulted in increase of both cholesterol and iron levels, while at $5 \mu \mathrm{M}$ it had no effect (Figure 2G-H).

Effect of iron chelators and cholesterol decreasing agents on neuroblastoma cells. To investigate whether iron chelators and/or inhibitors of cholesterol uptake or synthesis would 

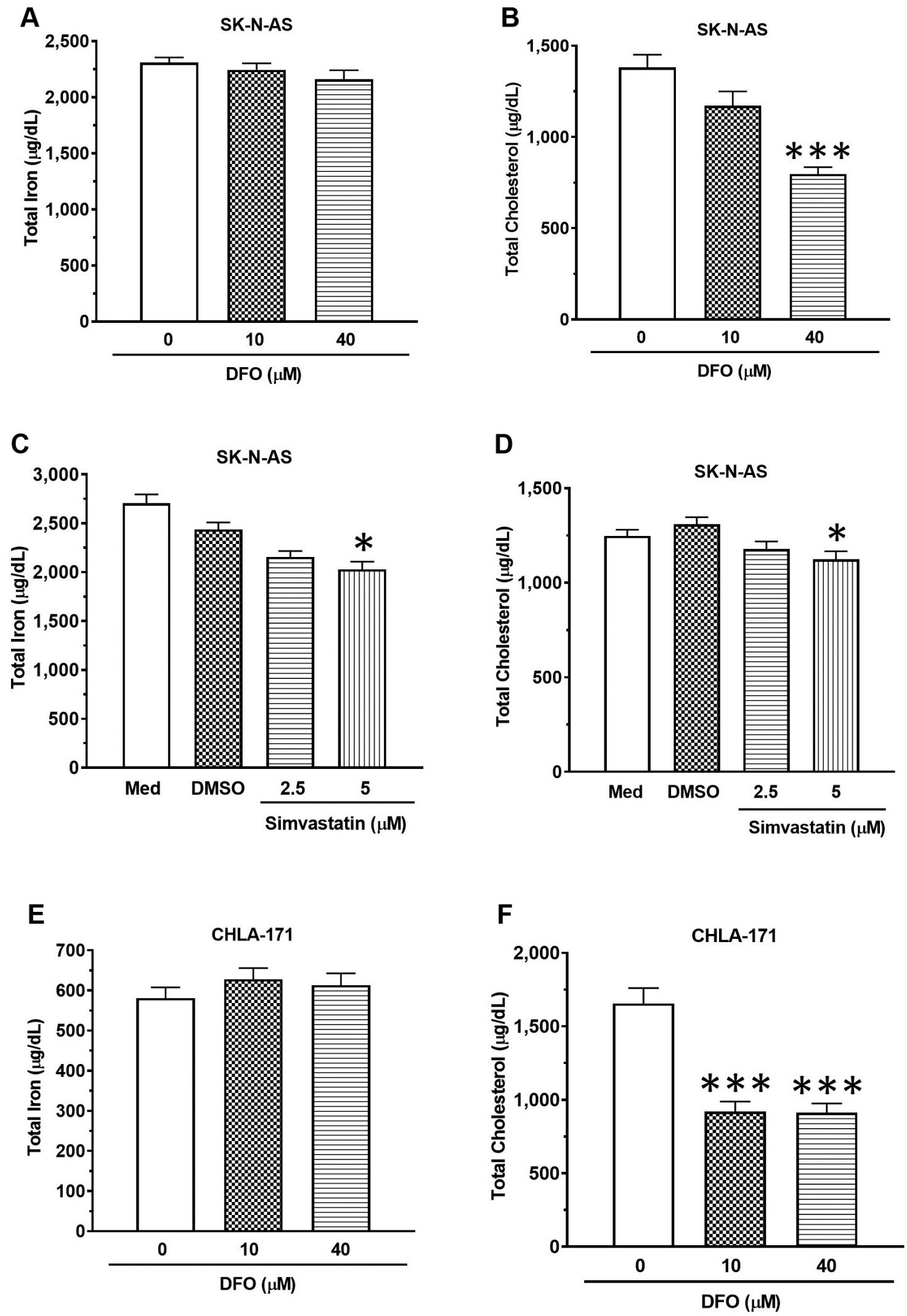

Figure 2. Continued 

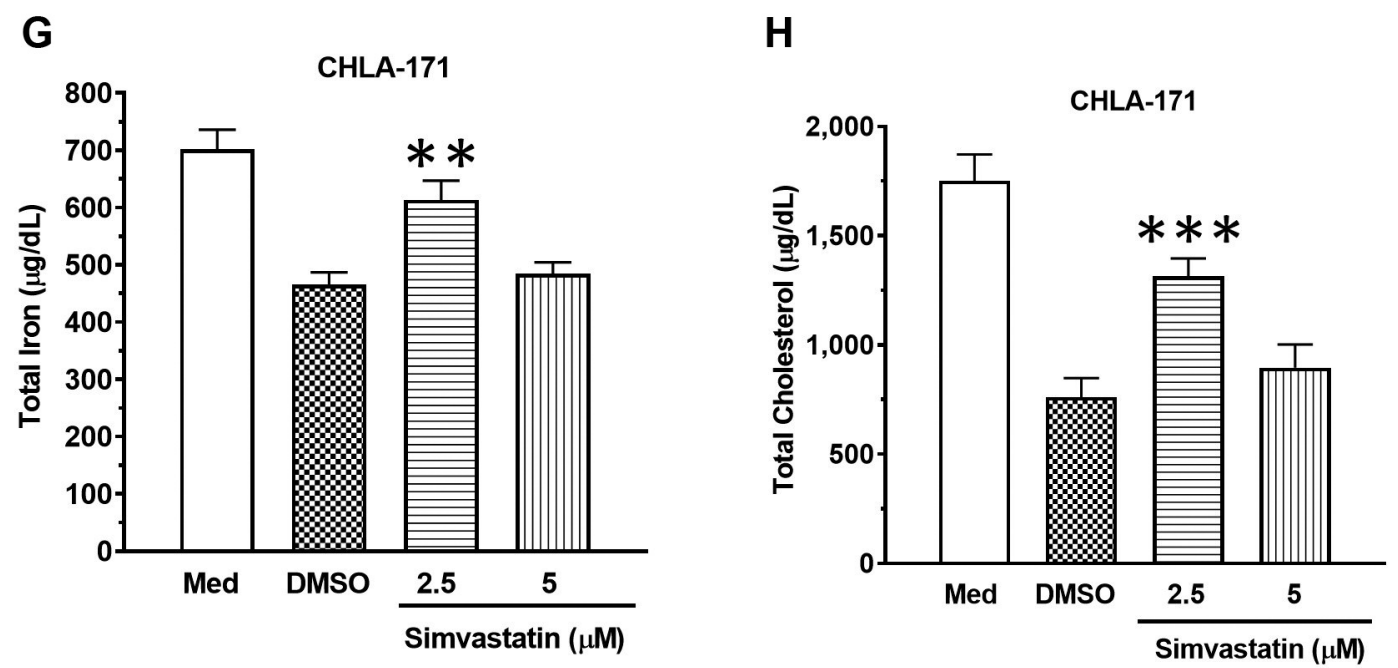

Figure 2. Effect of DFO or simvastatin on iron and cholesterol levels in SK-N-AS and CHLA-171 cells. SK-N-AS and CHLA-171 cells cultured in lipoprotein-deficient serum-containing medium were treated with vehicle or different doses of DFO (A-B, E-F) or simvastatin $(C-D, G-H)$ for two days prior to determination of iron or cholesterol. Intracellular iron and cholesterol contents were determined using the QuantiChrom Iron Assay Kit and Biovision cholesterol assay kit, respectively. Concentrations of total iron or cholesterol are given in $\mu \mathrm{g} / \mathrm{dl}$, as described in the legend of Figure 1. Results shown indicate the mean \pm SEM of three independent experiments. The asterisks indicate a significant difference compared to the control group. $* p<0.05, * * p<0.01, * * * p<0.001$.

inhibit the growth of neuroblastoma cells in vitro, we examined their effect on cell viability. When determining the effect of cholesterol decreasing agents, cells were maintained in medium containing $1 \%$ FBS in order to minimize the effect of serum cholesterol. SK-N-AS cells were found to be less susceptible to the iron chelator DFO (Figure 3A) but more sensitive to simvastatin, an inhibitor of HMGCoA reductase, than SH-SY5Y cells (Figure 3B). The response to ezetimibe, an inhibitor of cholesterol uptake by cells, was similar for both the SH-SY5Y and SK-N-AS cells (Figure 3C). Meanwhile, CHLA-171 cells were more sensitive to simvastatin than other drugs (Figure 3D). A comparison of the cytotoxicity of DFO with that of other iron chelators developed for cancer treatment indicated that it was less toxic to SK-N-AS and CHLA-171 cells (Figure 3E and F) than $\mathrm{Dp} 44 \mathrm{mT}$, triapine, and $\mathrm{DpC}$. Of the four iron chelators tested, $\mathrm{DpC}$ was most cytotoxic.

\section{Discussion}

The present study evaluated the relationship between iron and cholesterol in drug-resistant human neuroblastoma cells to determine whether altering either or both might provide a potential treatment strategy. Using known drug-resistant neuroblastoma cells, we found a partial association between cellular iron and cholesterol concentrations.

To study the relationship between iron and cholesterol metabolism, we focused on the drug-resistant SK-N-AS and
CHLA-171 cells. As far as we know, analyses of the levels of iron and cholesterol in neuroblastoma cells, especially those derived from tumors of drug-resistant patients, have not been previously reported. Although we hypothesized that drugresistant SK-N-AS and CHLA-171 cells would have increased iron and cholesterol, there was no apparent association between the level of iron/cholesterol and drug resistance in the cells studied. Instead, we observed that as iron levels increased, cholesterol levels decreased during their time in culture. These data indicate that neuroblastoma cells may take up iron for use during cell proliferation, while cholesterol accumulation may depend on its rate of usage during cell proliferation, availability in the culture medium, and rate of synthesis by the cells. Although there are several publications discussing either the role of iron or of cholesterol in cancer, those describing the effects of iron and cholesterol in cancer is limited. One study of the effects of iron described the interaction between ferroptosis (accumulation of irondependent lethal lipid peroxides) and lipid metabolism in cancer (35). The postulate drawn from their observations is that because cancer cells require higher levels of iron and lipid metabolism for rapid growth, they are more susceptible to ferroptosis. Excess iron is associated with cancer cell proliferation, migration, regulation of DNA replication and the cell cycle, as well as the tumor microenvironment $(36,37)$. Studies of the effect of iron on neuroblastoma and Ewing's sarcoma indicated that iron functioned in formation of $\mathrm{N}$ glycolyl neuraminic acid, a sugar found in sialylated 
A
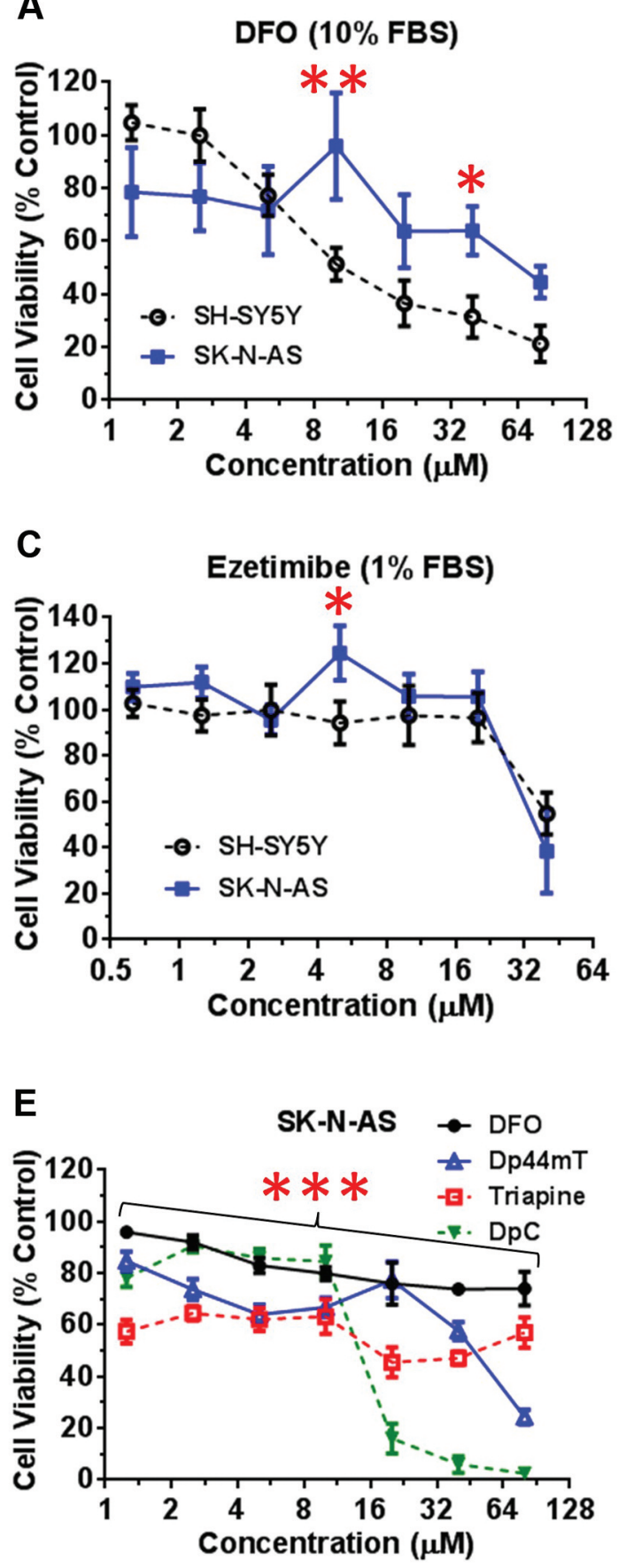

B

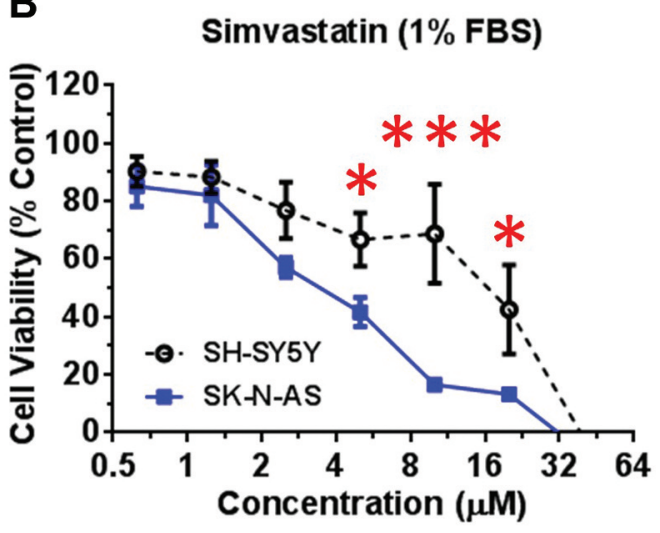

D
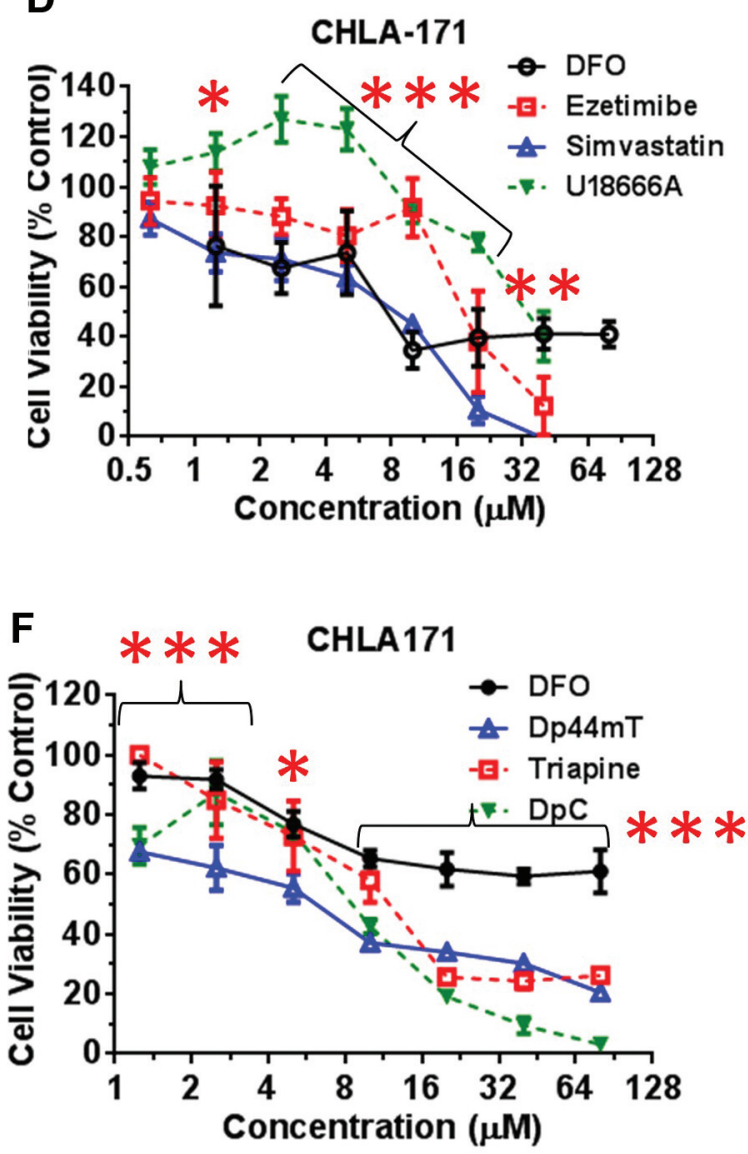

Figure 3. Cytotoxic effects of iron chelators and cholesterol decreasing agents on neuroblastoma cells. (A) Human neuroblastoma cells (SH-SY5Y, $S K-N-A S)$ were cultured overnight in complete cell culture medium prior to a 2-day exposure to the iron chelator DFO. (B-C) Human neuroblastoma cells (SH-SY5Y, SK-N-AS) were cultured overnight in complete cell culture medium prior to exposure to either simvastatin or ezetimibe, cholesterol decreasing agents, in medium containing 1\% FBS for 2 days. (D) Human neuroblastoma CHLA-171 cells were cultured overnight in complete cell culture medium prior to a 2-day exposure to either an iron chelator (DFO) or cholesterol decreasing agent (simvastatin, ezetimibe, or U18666A) in a medium containing $1 \%$ FBS. (E, F) Human neuroblastoma cells (SK-N-AS, CHLA-171) were cultured overnight in complete cell culture medium prior to a 2-day exposure to an iron chelator, either DFO, Dp44mT, triapine, or DpC. (A-F) In each instance after two days of exposure, cytotoxicity was ascertained using the alamarBlue assay. Results represent the means of three independent experiments with error bars corresponding to standard errors. For each plot, symbols indicate statistically significant differences between treatments at the indicated concentrations. ${ }^{*} p<0.05$, ** $<<0.01$, $* * * p<0.001$. 
glycosphingolipids in cancer, but not in healthy human tissue (38). In a cohort of the Framingham study it was found that high iron coupled with either a high level of very low-density lipoprotein cholesterol or a low level of high density lipoprotein cholesterol appeared to increase cancer risk (23). Therefore, our results and previous reports suggest that iron in conjunction with its effects on lipid metabolism plays a role in cancer development. Examples of how cholesterol and derivatives thereof might function in cancer cell progression include binding to sterol response element binding proteins, nuclear sterol receptors, lipid particle receptors $(39,40)$, and the formation of lipid rafts (glycolipid enriched microdomains) $(41,42)$, that can function in signal transduction and affect membrane fluidity. The finding that cholesterol and gangliosides are present in lipid rafts and the composition of the sialic acid present on gangliosides can be modified by the presence of iron, further supports the need for interrogation of how iron and cholesterol affect neuroblastoma behavior.

In this study, the iron chelator, DFO, did not cause a decrease in iron in the drug-resistant SK-N-AS and CHLA171 cells. This observation agrees with an earlier report that nonheme iron was not altered in iron treated hepatocytes cultured in media containing DFO (43). In contrast, Liu et al. found that DFO depleted intracellular iron in non-aggressive human breast cancer MCF-7 cells. At the same time, it significantly increased the intracellular iron level in aggressive breast cancer MDA-MB-231 cells (44). Therefore, failure of DFO treatment to alter iron levels in drug-resistant neuroblastoma cells may be due to i) activation of transferrin receptor-mediated or non-transferrin receptor-mediated iron uptake for the iron complement, ii) the difference between drug-sensitive cells and drug-resistant cells, iii) cell type (e.g., neuroblastomas vs. breast cancer). The current findings are based on two drug-resistant neuroblastomas (SK-N-AS and CHLA-171). To address this possibility, the effect of DFO treatment on iron levels of neuroblastoma cells should be assessed relative to its effect on other types of cancer cells. While DFO did not affect iron content, it did result in a decreased cholesterol level in both SK-N-AS and CHLA-171 cells. This observation, coupled with the finding that iron level was decreased in SK-N-AS cells by the HMGCoA inhibitor simvastatin, indicates that a bi-directional association between iron and cholesterol may exist in human neuroblastoma SK-N-AS cells. The bi-directional association between iron and cholesterol appears to be cell dependent since it was not seen in CHLA-171 cells.

Previously, we reported that C282Y HFE/SH-SY5Y neuroblastoma cells showed resistance to chemotherapy and radiation treatment (45), but were significantly more sensitive to the iron chelator DFO than WT HFE/SH-SY5Y cells (28). These observations coupled with the observation that C282Y HFE/SH-SY5Y cells were more sensitive to U18666A than WT HFE/SH-SY5Y cells (27) supported interrogation of whether iron chelation therapy and cholesterol decreasing agents could be effective against drugresistant neuroblastomas. The finding that DFO had a mild cytotoxic effect, but did not completely kill drug-resistant neuroblastoma SK-N-AS cells agrees with the observation that DFO did not have anti-tumor effects in either the IMR32 or JBN-1 neuroblastoma xenograft nude mouse model (46). Iron chelators have antioxidant and free radical scavenging activity (47). Both functions are essential for cancer treatment. As expected from the effect of iron chelators, a potent antioxidant N-acetyl-L-cysteine (NAC) protected SH-SY5Y cells from oxidative stress and cell cytotoxicity (48).

Among the tested iron chelators, DpC had the strongest cytotoxic effect on the SK-N-AS and CHLA-171 cells, while simvastatin was the most cytotoxic of the cholesteroldecreasing agents tested. The later result suggests that the ratelimiting enzyme (HMG-CoA reductase) of cholesterol synthesis is critical for survival of drug-resistant neuroblastoma cells. The anti-tumor effect of $\mathrm{DpC}$ on a neuroblastoma animal model (orthotopic SK-N-LP/Luciferase xenograft) has been reported (49). Although $M Y C N$ amplification is observed in about $30 \%$ of high-risk neuroblastomas (4), other high-risk neuroblastomas lacking $M Y C N$ amplification such as those caused by SK-N-AS cells also acquire multidrug resistance. The SK-N-AS cells express higher levels of multidrug resistance-related protein 1 and sphingolipid than SK-N-DZ cells (50). The SK-N-AS cells also maintain drug resistance through overexpression of neuronal apoptosis inhibitory protein (51). While both CHLA-136 and CHLA-171 cells are multidrug resistant, p53 is functional in CHLA-136 cells, but not in CHLA-171 cells (24). These data support the possibility that alterations in iron and cholesterol metabolism are part of the cell's drug resistance mechanism, similar to other factors (MYCN amplification, multidrug resistance-related protein 1, functional p53 expression) identified in neuroblastomas.

The results obtained by studying several established neuroblastoma cell lines to ascertain the association between iron and cholesterol underscore the variability seen in the response of neuroblastomas to different therapeutic agents as well as the need for further studies. For example, in addition to testing the efficacy of other statins and iron chelators, and combinations of iron chelators and cholesterol decreasing agents, studies should be done to ascertain the most effective approach to treat neuroblastomas. The finding that the cell lines have different doubling times and optimum cell culture conditions as well as more subtle differences related to the tumors from which they were derived may have influenced our results. Therefore, to determine whether an association exists between iron and cholesterol and whether it is found in the majority of human neuroblastomas an extended study of multiple cell lines derived from different human neuroblastomas is needed. Such studies could monitor the 
efficacy of other iron chelators (e.g., Dp44mT) and other cholesterol decreasing agents (e.g., lovastatin) alone and together on cells in culture. Those that seem promising would then be tested on multiple in vivo neuroblastoma tumor models. While possible molecular mechanisms for the effects of both iron and cholesterol on cancer development are discussed above, assays to determine which one(s) cause the significant effect(s) found in multiple neuroblastoma cell lines should be done. For example, the effect of iron and cholesterol alteration on cell signaling pathways, and expression of proteins needed for lipid synthesis could be assessed.

In summary, our results indicate that drug-resistant neuroblastoma cells may have a diverse pattern of iron and cholesterol levels. There is a bi-directional association between iron and cholesterol in human neuroblastoma SK-N-AS cells. The results of in vitro studies of human neuroblastoma cells indicated that they were susceptible to iron chelators and cholesterol inhibitors, however, further investigation is needed to ascertain whether alteration of the tumors' iron and/or cholesterol metabolism can be used to enhance treatment of neuroblastomas refractory to approaches currently in use.

\section{Conflicts of Interest}

The Authors declare no conflicts of interest.

\section{Authors' Contributions}

Conceptualization: Sang Y Lee; Literature search and analysis: Sang Y Lee, Cara-Lynne Schengrund, Junjia Zhu; Data generation: Sang Y Lee, Becky Slagle-Webb; Writing - original draft preparation: Sang Y Lee; Writing - review and editing: Sang Y Lee, Becky Slagle-Webb, Cara-Lynne Schengrund, Junjia Zhu, James R Connor.

\section{Acknowledgements}

We thank Dr. Barbara Miller for kindly providing the SK-N-AS human neuroblastoma cells that she had obtained from the ATCC. We thank the Children's Oncology Group for CHLA-136 and CHLA-171 cells. The Authors thank Mrs. Sara Langan for her critical reading of this manuscript.

\section{Funding}

This research was funded by CHILDREN'S MIRACLE NETWORK (PENN STATE HERSHEY MEDICAL CENTER, grant number 417-20-HY 24NB).

\section{References}

1 National Cancer Institute. Surveillance, epidemiology and end results database. Available at: http://seer.cancer.gov [Last accessed on April 15, 2021]

2 Joshi S: Targeting the tumor microenvironment in neuroblastoma: recent advances and future directions. Cancers (Basel) 12(8): 2057, 2020. PMID: 32722460. DOI: 10.3390/cancers 12082057
3 Tolbert VP and Matthay KK: Neuroblastoma: clinical and biological approach to risk stratification and treatment. Cell Tissue Res 372(2): 195-209, 2018. PMID: 29572647. DOI: 10.1007/s00441-018-2821-2

4 Matthay KK, Maris JM, Schleiermacher G, Nakagawara A, Mackall CL, Diller L and Weiss WA: Neuroblastoma. Nat Rev Dis Primers 2: 16078, 2016. PMID: 27830764. DOI: 10.1038/nrdp.2016.78

5 Tomolonis JA, Agarwal S and Shohet JM: Neuroblastoma pathogenesis: deregulation of embryonic neural crest development. Cell Tissue Res 372(2): 245-262, 2018. PMID: 29222693. DOI: $10.1007 / \mathrm{s} 00441-017-2747-0$

6 Shimada H, Ambros IM, Dehner LP, Hata J, Joshi VV, Roald B, Stram DO, Gerbing RB, Lukens JN, Matthay KK and Castleberry RP: The international neuroblastoma pathology classification (the Shimada system). Cancer 86(2): 364-372, 1999. PMID: 10421273.

7 Cohn SL, Pearson AD, London WB, Monclair T, Ambros PF, Brodeur GM, Faldum A, Hero B, Iehara T, Machin D, Mosseri V, Simon T, Garaventa A, Castel V, Matthay KK and INRG Task Force: The International Neuroblastoma Risk Group (INRG) classification system: an INRG Task Force report. J Clin Oncol 27(2): 289-297, 2009. PMID: 19047291. DOI: 10.1200/JCO. 2008.16.6785

8 Schönherr C, Ruuth K, Kamaraj S, Wang CL, Yang HL, Combaret V, Djos A, Martinsson T, Christensen JG, Palmer RH and Hallberg B: Anaplastic Lymphoma Kinase (ALK) regulates initiation of transcription of MYCN in neuroblastoma cells. Oncogene 31(50): 5193-5200, 2012. PMID: 22286764. DOI: 10.1038/onc.2012.12

9 Tucker E, Poon E and Chesler L: Targeting MYCN and ALK in resistant and relapsing neuroblastoma. Cancer Drug Resistance, 2019. DOI: $10.20517 / \mathrm{cdr} .2019 .009$

10 Cao Y, Jin Y, Yu J, Wang J, Yan J and Zhao Q: Research progress of neuroblastoma related gene variations. Oncotarget 8(11): 18444-18455, 2017. PMID: 28055978. DOI: 10.18632/ oncotarget.14408

11 Zhuo ZJ, Liu W, Zhang J, Zhu J, Zhang R, Tang J, Yang T, Zou $\mathrm{Y}, \mathrm{He} \mathrm{J}$ and Xia H: Functional polymorphisms at ERCC1/XPF genes confer neuroblastoma risk in Chinese children. EBioMedicine 30: 113-119, 2018. PMID: 29544698. DOI: 10.1016/j.ebiom.2018.03.003

12 Zhuo Z, Zhou C, Fang Y, Zhu J, Lu H, Zhou H, Wu H, Wang Y and $\mathrm{He} \mathrm{J}$ : Correlation between the genetic variants of base excision repair (BER) pathway genes and neuroblastoma susceptibility in eastern Chinese children. Cancer Commun (Lond) 40(11): 641-646, 2020. PMID: 32780923. DOI: $10.1002 / \mathrm{cac} 2.12088$

13 Barr EK and Applebaum MA: Genetic predisposition to neuroblastoma. Children (Basel) 5(9): 119, 2018. PMID: 30200332. DOI: $10.3390 /$ children5090119

14 Fonseca-Nunes A, Jakszyn P and Agudo A: Iron and cancer risk - a systematic review and meta-analysis of the epidemiological evidence. Cancer Epidemiol Biomarkers Prev 23(1): 12-31, 2014. PMID: 24243555. DOI: 10.1158/10559965.EPI-13-0733

15 Wen CP, Lee JH, Tai YP, Wen C, Wu SB, Tsai MK, Hsieh DP, Chiang HC, Hsiung CA, Hsu CY and Wu X: High serum iron is associated with increased cancer risk. Cancer Res 74(22): 65896597, 2014. PMID: 25228650. DOI: 10.1158/0008-5472.CAN$14-0360$ 
16 Graham RM, Chua AC, Carter KW, Delima RD, Johnstone D, Herbison CE, Firth MJ, O'Leary R, Milward EA, Olynyk JK and Trinder D: Hepatic iron loading in mice increases cholesterol biosynthesis. Hepatology 52(2): 462-471, 2010. PMID: 20683946. DOI: $10.1002 /$ hep. 23712

17 Krycer JR and Brown AJ: Cholesterol accumulation in prostate cancer: a classic observation from a modern perspective. Biochim Biophys Acta 1835(2): 219-229, 2013. PMID: 23357067. DOI: 10.1016/j.bbcan.2013.01.002

18 Pelton K, Freeman MR and Solomon KR: Cholesterol and prostate cancer. Curr Opin Pharmacol 12(6): 751-759, 2012. PMID: 22824430. DOI: 10.1016/j.coph.2012.07.006

19 Kolanjiappan K, Ramachandran CR and Manoharan S: Biochemical changes in tumor tissues of oral cancer patients. Clin Biochem 36(1): 61-65, 2003. PMID: 12554062. DOI: 10.1016/s0009-9120(02)00421-6

20 Nakamura K, Hongo A, Kodama J and Hiramatsu Y: Fat accumulation in adipose tissues as a risk factor for the development of endometrial cancer. Oncol Rep 26(1): 65-71, 2011. PMID: 21491090. DOI: 10.3892/or.2011.1259

21 Rockfield S, Chhabra R, Robertson M, Rehman N, Bisht R and Nanjundan M: Links between iron and lipids: implications in some major human diseases. Pharmaceuticals (Basel) 11(4): 113, 2018. PMID: 30360386. DOI: 10.3390/ph11040113

22 Wells BJ, Mainous AG 3rd, Everett CJ and Gill JM: Iron, cholesterol, and the risk of cancer in an 18-year cohort. Asian Pac J Cancer Prev 6(4): 505-509, 2005. PMID: 16436001.

23 Mainous AG 3rd, Wells BJ, Koopman RJ, Everett CJ and Gill JM: Iron, lipids, and risk of cancer in the Framingham Offspring cohort. Am J Epidemiol 161(12): 1115-1122, 2005. PMID: 15937020. DOI: 10.1093/aje/kwi131

24 Keshelava N, Tsao-Wei D and Reynolds CP: Pyrazoloacridine is active in multidrug-resistant neuroblastoma cell lines with nonfunctional p53. Clin Cancer Res 9(9): 3492-3502, 2003. PMID: 12960142.

25 Keshelava N, Davicioni E, Wan Z, Ji L, Sposto R, Triche TJ and Reynolds CP: Histone deacetylase 1 gene expression and sensitization of multidrug-resistant neuroblastoma cell lines to cytotoxic agents by depsipeptide. J Natl Cancer Inst 99(14): 1107-1119, 2007. PMID: 17623797. DOI: 10.1093/jnci/djm044

26 Sudarshan S, Sourbier C, Kong HS, Block K, Valera Romero VA, Yang Y, Galindo C, Mollapour M, Scroggins B, Goode N, Lee MJ, Gourlay CW, Trepel J, Linehan WM and Neckers L: Fumarate hydratase deficiency in renal cancer induces glycolytic addiction and hypoxia-inducible transcription factor 1alpha stabilization by glucose-dependent generation of reactive oxygen species. Mol Cell Biol 29(15): 4080-4090, 2009. PMID: 19470762. DOI: 10.1128/MCB.00483-09

27 Ali-Rahmani F, Huang MA, Schengrund CL, Connor JR and Lee SY: C282Y-HFE gene variant affects cholesterol metabolism in human neuroblastoma cells. PLoS One 9(2): e88724, 2014. PMID: 24533143. DOI: 10.1371/journal.pone.0088724

28 Lee SY, Patton SM, Henderson RJ and Connor JR: Consequences of expressing mutants of the hemochromatosis gene (HFE) into a human neuronal cell line lacking endogenous HFE. FASEB J 21(2): 564-576, 2007. PMID: 17194693. DOI: 10.1096/fj.06-6397com

29 Wong WW, Dimitroulakos J, Minden MD and Penn LZ: HMGCoA reductase inhibitors and the malignant cell: the statin family of drugs as triggers of tumor-specific apoptosis. Leukemia 16(4): 508-519, 2002. PMID: 11960327. DOI: 10.1038/sj.leu.2402476
30 Lui GY, Kovacevic Z, Richardson V, Merlot AM, Kalinowski DS and Richardson DR: Targeting cancer by binding iron: Dissecting cellular signaling pathways. Oncotarget 6(22): 1874818779, 2015. PMID: 26125440. DOI: 10.18632/oncotarget.4349

31 Sparrow SM, Carter JM, Ridgway ND, Cook HW and Byers DM: U18666A inhibits intracellular cholesterol transport and neurotransmitter release in human neuroblastoma cells. Neurochem Res 24(1): 69-77, 1999. PMID: 9973239. DOI: 10.1023/a:1020932130753

32 Xie C, Zhou ZS, Li N, Bian Y, Wang YJ, Wang LJ, Li BL and Song BL: Ezetimibe blocks the internalization of NPC1L1 and cholesterol in mouse small intestine. J Lipid Res 53(10): 20922101, 2012. PMID: 22811412. DOI: 10.1194/jlr.M027359

33 Chan J, Kushwaha RS, Vandeberg JF and Vandeberg JL: Effect of ezetimibe on plasma cholesterol levels, cholesterol absorption, and secretion of biliary cholesterol in laboratory opossums with high and low responses to dietary cholesterol. Metabolism 57(12): 16451654, 2008. PMID: 19013286. DOI: 10.1016/j.metabol.2008.07.019

34 Sankaranarayanan S, de la Llera-Moya M, Drazul-Schrader D, Asztalos BF, Weibel GL and Rothblat GH: Importance of macrophage cholesterol content on the flux of cholesterol mass. J Lipid Res 51(11): 3243-3249, 2010. PMID: 20713652. DOI: 10.1194/jlr.M008441

$35 \mathrm{Li} \mathrm{D}$ and Li Y: The interaction between ferroptosis and lipid metabolism in cancer. Signal Transduct Target Ther 5(1): 108, 2020. PMID: 32606298. DOI: 10.1038/s41392-020-00216-5

36 Wang Y, Yu L, Ding J and Chen Y: Iron metabolism in cancer. Int J Mol Sci 20(1): 95, 2018. PMID: 30591630. DOI: 10.3390/ijms20010095

37 Brown RAM, Richardson KL, Kabir TD, Trinder D, Ganss R and Leedman PJ: Altered iron metabolism and impact in cancer biology, metastasis, and immunology. Front Oncol 10: 476, 2020. PMID: 32328462. DOI: 10.3389/fonc.2020.00476

38 Scursoni AM, Galluzzo L, Camarero S, Lopez J, Lubieniecki F, Sampor C, Segatori VI, Gabri MR, Alonso DF, Chantada G and de Dávila MT: Detection of N-glycolyl GM3 ganglioside in neuroectodermal tumors by immunohistochemistry: an attractive vaccine target for aggressive pediatric cancer. Clin Dev Immunol 2011: 245181, 2011. PMID: 21941577. DOI: $10.1155 / 2011 / 245181$

39 Huang B, Song BL and Xu C: Cholesterol metabolism in cancer: mechanisms and therapeutic opportunities. Nat Metab 2(2): 132141, 2020. PMID: 32694690. DOI: 10.1038/s42255-020-0174-0

40 Gabitova L, Gorin A and Astsaturov I: Molecular pathways: sterols and receptor signaling in cancer. Clin Cancer Res 20(1): 28-34, 2014. PMID: 24158702. DOI: 10.1158/1078-0432.CCR13-0122

41 Simons K and Ikonen E: Functional rafts in cell membranes. Nature 387(6633): 569-572, 1997. PMID: 9177342. DOI: 10.1038/42408

42 Ohmi Y, Ohkawa Y, Yamauchi Y, Tajima O, Furukawa K and Furukawa K: Essential roles of gangliosides in the formation and maintenance of membrane microdomains in brain tissues. Neurochem Res 37(6): 1185-1191, 2012. PMID: 22488331. DOI: $10.1007 / \mathrm{s} 11064-012-0764-7$

43 Trinder D, Batey RG, Morgan EH and Baker E: Effect of cellular iron concentration on iron uptake by hepatocytes. Am J Physiol 259(4 Pt 1): G611-G617, 1990. PMID: 2221071. DOI: 10.1152/ajpgi.1990.259.4.G611

44 Liu P, He K, Song H, Ma Z, Yin W and Xu LX: Deferoxamineinduced increase in the intracellular iron levels in highly aggressive breast cancer cells leads to increased cell migration 
by enhancing TNF- $\alpha$-dependent NF- $x \mathrm{~B}$ signaling and TGF- $\beta$ signaling. J Inorg Biochem 160: 40-48, 2016. PMID: 27138103. DOI: $10.1016 /$ j.jinorgbio.2016.04.014

45 Lee SY, Liu S, Mitchell RM, Slagle-Webb B, Hong YS, Sheehan JM and Connor JR: HFE polymorphisms influence the response to chemotherapeutic agents via induction of p16INK4A. Int $\mathrm{J}$ Cancer 129(9): 2104-2114, 2011. PMID: 21190189. DOI: 10.1002/ijc. 25888

46 Selig RA, White L, Gramacho C, Sterling-Levis K, Fraser IW and Naidoo D: Failure of iron chelators to reduce tumor growth in human neuroblastoma xenografts. Cancer Res 58(3): 473-478, 1998. PMID: 9458092.

47 Adjimani JP and Asare P: Antioxidant and free radical scavenging activity of iron chelators. Toxicol Rep 2: 721-728, 2015. PMID: 28962407. DOI: 10.1016/j.toxrep.2015.04.005

48 Jurkowska $\mathrm{H}$ and Wróbel M: Inhibition of human neuroblastoma cell proliferation by $\mathrm{N}$-acetyl-L-cysteine as a result of increased sulfane sulfur level. Anticancer Res 38(9): 5109-5113, 2018. PMID: 30194156. DOI: 10.21873/anticanres.12831

49 Guo ZL, Richardson DR, Kalinowski DS, Kovacevic Z, Tan-Un KC and Chan GC: The novel thiosemicarbazone, di-2pyridylketone 4-cyclohexyl-4-methyl-3-thiosemicarbazone $(\mathrm{DpC})$, inhibits neuroblastoma growth in vitro and in vivo via multiple mechanisms. J Hematol Oncol 9(1): 98, 2016. PMID: 27678372. DOI: 10.1186/s13045-016-0330-x
50 Dijkhuis AJ, Douwes J, Kamps W, Sietsma H and Kok JW: Differential expression of sphingolipids in P-glycoprotein or multidrug resistance-related protein 1 expressing human neuroblastoma cell lines. FEBS Lett 548(1-3): 28-32, 2003. PMID: 12885402. DOI: 10.1016/s0014-5793(03)00721-x

51 Harvey H, Piskareva O, Creevey L, Alcock LC, Buckley PG, O'Sullivan MJ, Segura MF, Gallego S, Stallings RL and Bray IM: Modulation of chemotherapeutic drug resistance in neuroblastoma SK-N-AS cells by the neural apoptosis inhibitory protein and miR-520f. Int J Cancer 136(7): 1579-1588, 2015. PMID: 25137037. DOI: 10.1002/ijc.29144
Received April 17, 2021

Revised May 8, 2021

Accepted May 19, 2021 